\title{
Some More Results on Total Equitable Bondage Number of A Graph
}

\author{
S. K. Vaidya ${ }^{1 *}$, A. D. Parmar ${ }^{2}$ \\ ${ }^{1}$ Department of Mathematics, Saurashtra University, Rajkot-360005, India \\ ${ }^{2}$ Atmiya Institute of Technology \& Science for Diploma Studies \\ Rajkot-360005, India
}

Received 13 March 2019, accepted in final revised form 26 May 2019

\begin{abstract}
The bondage number $b(G)$ of a nonempty graph $G$ is the minimum cardinality among all sets of edges $E_{0} \subseteq E(G)$ for which $\gamma\left(G-E_{0}\right)>\gamma(G)$. An equitable dominating set $D$ is called a total equitable dominating set if the induced subgraph $\langle D\rangle$ has no isolated vertices. The total equitable domination number $\gamma_{t}^{e}(G)$ of $G$ is the minimum cardinality of a total equitable dominating set of $G$. If $\gamma_{t}^{e}(G) \neq|V(G)|$ and $\left\langle G-E_{0}>\right.$ contains no isolated vertices then the total equitable bondage number $b_{t}^{e}(G)$ of a graph $G$ is the minimum cardinality among all sets of edges $E_{0} \subseteq E(G)$ for which $\gamma_{t}^{e}\left(G-E_{0}\right)>\gamma_{t}^{e}(G)$. In the present work we prove some characterizations and investigate total equitable bondage number of Ladder and degree splitting of path.
\end{abstract}

Keywords: Dominating set; Equitable dominating set; Total dominating set; Bondage number.

(C) 2019 JSR Publications. ISSN: 2070-0237 (Print); 2070-0245 (Online). All rights reserved. doi: http://dx.doi.org/10.3329/jsr.v11i3.40573

J. Sci. Res. 11 (3), 303-309 (2019)

\section{Introduction}

We begin with simple, finite and undirected graph $G=(V(G), E(G))$. We denote the degree of a vertex $v$ in a graph $G$ by $d_{G}(v)$. The maximum degree among all the vertices of $G$ is denoted by $\Delta(G)$. For any real number $n,\lceil n\rceil$ denotes the smallest integer not less than $n$ and $\lfloor n\rfloor$ denotes the greatest integer not greater than $n$.

The domination in graph is one of the fastest growing concepts in graph theory. Many variants of domination models are available in literature: Restrained Domination, Equitable Domination, Total Domination and Total Equitable Domination are among worth to mention.

Definition 1.1: A set $D \subseteq V(G)$ of vertices in a graph $G$ is called dominating set if every vertex $v \in V(G)$ is either an element of $D$ or is adjacent to an element of $D$. The minimum cardinality of a dominating set is called the domination number of $G$ which is denoted by $\gamma(G)$.

*Corresponding author: samirkvaidya@yahoo.co.in 
Definition 1.2: The bondage number $b(G)$ of a nonempty graph $G$ is the minimum cardinality among all sets of edges $E_{0} \subseteq E(G)$ for which $\gamma\left(G-E_{0}\right)>\gamma(G)$ [1] .

The concept of bondage number was introduced by Fink et al. [1] which is useful for measuring the vulnerability of the network under link failure. Variety of bondage numbers are introduced on the basis of characteristic of dominating sets. Some of them are total bondage number, equitable bondage number, total equitable bondage number are really noteworthy.

Definition 1.3: A subset $D$ of $V(G)$ is called an equitable dominating set if for every $v \in V(G)-D$ there exists a vertex $u \in D$ such that $u v \in E(G)$ and $\left|d_{G}(v)-d_{G}(u)\right| \leq$ 1 [2]. The minimum cardinality of $D$ is called the equitable domination number of $G$ which is denoted by $\gamma^{e}(G)$.

Definition 1.4: An equitable bondage number $b^{e}(G)$ of a graph $G$ is the cardinality of a smallest set $E_{0} \subseteq E(G)$ of edges for which $\gamma^{e}\left(G-E_{0}\right)>\gamma^{e}(G)$ [3].

The concept of equitable domination was introduced by Swaminathan and Dharmalingam [2] while the concept of equitable bondage number was introduced by Deepak et al. [3].

Definition 1.5: A subset $D$ of $V(G)$ is called a total dominating set of $G$ if $N(D)=V(G)$ or equivalently if every vertex $v \in V(G)$ is adjacent to at least one element in $D$ [4]. The minimum cardinality of total dominating set is called total domination number which is denoted by $\gamma_{t}(G)$.

Definition 1.6: If $\gamma_{t} \neq|V(G)|$ and $\left\langle G-E_{0}>\right.$ contains no isolated vertices then the total bondage number $b_{t}(G)$ of a graph $G$ is the minimum cardinality among all sets of edges $E_{0} \subseteq E(G)$ for which $\gamma_{t}\left(G-E_{0}\right)>\gamma_{t}(G)[5]$.

The concept of total domination was introduced by Cockayne et al. [4] while the concept of total bondage number was introduced by Kulli and Patwari [5].

Definition 1.7: An equitable dominating set $D$ is called a total equitable dominating set if the induced subgraph $\langle D\rangle$ has no isolated vertices [6]. The total equitable domination number $\gamma_{t}^{e}(G)$ of $G$ is the minimum cardinality of a total equitable dominating set of $G$.

The concepts of total dominating set and total equitable dominating set are useful for the formation of any committee. It is desirable that each committee member might feel comfortable knowing at least one member of the committee. In this situation, total domination is useful while there is no difference of opinion between any two members or they differ on at most one issue then the concept of equitable domination is applicable. A concept with both the blends is termed as total equitable dominating set was introduced by Basavanagoud et al. [6] and further formalized by Vaidya and Parmar [7-10]. Using the framework of total equitable dominating sets, we have introduced a new concept called total equitable bondage number [11]. 
The total equitable bondage number is a bondage number with the additional property that removal of an edge subset from the given graph results in a graph with larger total equitable domination number. This concept is also useful for measuring the link failure of network.

Definition 1.8: If $\gamma_{t}^{e}(G) \neq|V(G)|$ and $<G-E_{0}>$ contains no isolated vertices then the total equitable bondage number $b_{t}^{e}(G)$ of a graph $G$ is the minimum cardinality among all sets of edges $E_{0} \subseteq E(G)$ for which $\gamma_{t}^{e}\left(G-E_{0}\right)>\gamma_{t}^{e}(G)$ [11].

In the following Fig.1(a) for the graph $G, \gamma_{t}^{e}(G)=2$ but $\gamma_{t}^{e}(G-e)=3$ as shown in Fig. 1(b). Thus $b_{t}^{e}(G)=1$. The vertices of the respective sets are shown by solid vertices.

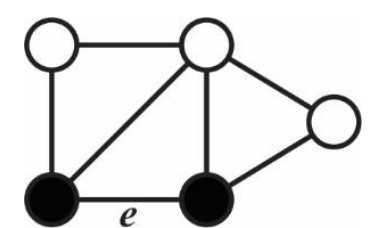

Fig. $1(\mathrm{a}) \cdot \gamma_{t}^{e}(G)=2$

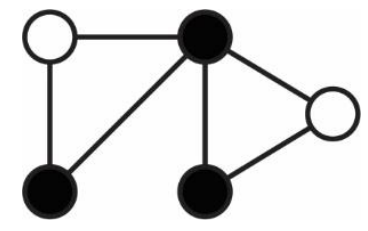

Fig. 1(b). $\gamma_{t}^{e}(G-e)=3$

We also introduce the concept of total equitable domination edge critical graph as follows.

Definition 1.9: A graph $G$ is called total equitable domination edge critical or $\gamma_{t}^{e}$-critical graph if the removal of any edge in the graph changes the total equitable domination number. i.e. $\gamma_{t}^{e}(G-e) \neq \gamma_{t}^{e}(G)$ for every edge $e \in E(G)$.

In the following Fig. 2(a) for the graph $G, \gamma_{t}^{e}(G)=8$ but $\gamma_{t}^{e}(G-e)=9$ as shown in Fig. 1(b). Thus $\gamma_{t}^{e}(G)=8 \neq 9=\gamma_{t}^{e}(G-e)$. Therefore $G$ is $\gamma_{t}^{e}$ - critical graph. The vertices of the respective sets are shown by solid vertices.

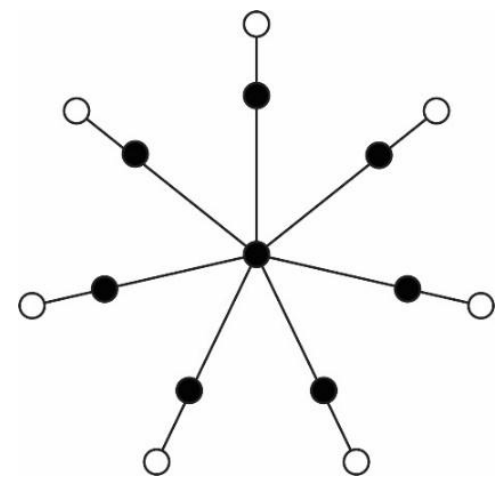

Fig. 2(a). $\gamma_{t}^{e}(G)=8$

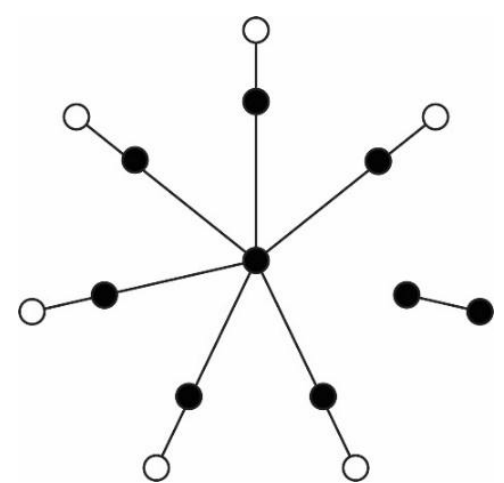

Fig. 2(b). $\gamma_{t}^{e}(G-e)=9$

Definition 1.10: The Cartesian product of $G$ and $H$ is a graph, denoted as $G \times H$ whose vertex set $V(G) \times V(H)$ [12]. Two vertices $(g, h)$ and $\left(g^{\prime}, h^{\prime}\right)$ are adjacent precisely if 
$g=g^{\prime}$ and $h h^{\prime} \in E(H)$ or $g g^{\prime} \in E(G)$ and $h=h^{\prime}$. Thus $V(G \times H)=\{(g, h) / g \in$ $V(G)$ and $h \in V(H)\} \quad$ and $E(G \times H)=\left\{(g, h)\left(g^{\prime}, h^{\prime}\right) / g=g^{\prime}, h h^{\prime} \in E(H)\right.$ or $g g^{\prime} \in$ $\left.E(G), h=h^{\prime}\right\}$.

Definition 1.11: The ladder $L_{n}$ is defined as $P_{n} \times P_{2}$ [12].

Definition 1.12: Let $G=(V(G), E(G))$ be a graph with $V(G)=S_{1} \cup S_{2} \cup S_{3} \cup \ldots \cup S_{t} \cup$ $T$, where each $S_{i}$ is a set of all the vertices having same degree (at least 2 vertices) and $T=V(G) \backslash \bigcup_{i=1}^{t} S_{i}$ [13]. The degree splitting graph $D S(G)$ is obtained from $G$ by adding vertices $w_{1}, w_{2}, w_{3}, \ldots, w_{t}$ and joining to each vertex of $S_{i}$ for $1 \leq i \leq t$.

Proposition 1.13: For any $k$-regular or $(k, k+1)$-biregular graph $G, \gamma_{t}(G)=$ $\gamma_{t}^{e}(G)[6]$.

Proposition 1.14: For any $k$-regular or $(k, k+1)$-biregular graph $G, b(G)=$ $b^{e}(G)[2]$.

Proposition 1.15: $\gamma_{t}\left(P_{n} \times P_{2}\right)=\gamma_{t}^{e}\left(P_{n} \times P_{2}\right)=2\left\lceil\frac{n}{3}\right\rceil[9]$.

Proposition 1.16: $b_{t}\left(L_{n}\right)= \begin{cases}1 & ; \text { if } n \equiv 0(\bmod 3) \\ 2 & ; \text { if } n \equiv 2(\bmod 3) \\ 3 & ; \text { otherwise }\end{cases}$

Proposition 1.17: $\gamma_{t}\left(D S\left(P_{n}\right)\right)=\left\{\begin{array}{lc}1 ; & \text { if } n=4 \\ 2 ; & \text { otherwise }\end{array}[8]\right.$

For the various graph theoretic notation and terminology we follow West [15] while for any undefined terms related to the concept of domination we refer to Haynes et al. [16].

Here we contribute some characterizations and also investigate total equitable bondage number of some graph families.

\section{Main Results}

Theorem 2.1: Let $G$ be any graph with $\gamma_{t}(G) \neq|V(G)|$ and $\gamma_{t}^{e}(G) \neq|V(G)|$. Let $E_{0}$ be a total bondage set with minimum cardinality. If $G-E_{0}$ be $k$-regular or $(k, k+$ 1) -biregular then $b_{t}(G)=b_{t}^{e}(G)$.

Proof: Let $G$ be any graph with $\gamma_{t}(G) \neq|V(G)|$ and $\gamma_{t}^{e}(G) \neq|V(G)|$. Let $E_{0}$ be a total bondage set of $G$ with minimum cardinality. If $G-E_{0}$ be $k$-regular or $(k, k+$ 1) -biregular then from Proposition 1.13, $\gamma_{t}\left(G-E_{0}\right)=\gamma_{t}^{e}\left(G-E_{0}\right)$. Hence, $b_{t}(G)=$ $b_{t}^{e}(G)$.

We have an immediate corollary as follows:

Corollary 2.2: Let $G$ be a graph. Let $E_{0}$ be a bondage set with minimum cardinality. If $G-E_{0}$ be $k$-regular or $(k, k+1)$-biregular then $b(G)=b^{e}(G)$. 
Proof: Let $G$ be a graph. Let $E_{0}$ be a bondage set with minimum cardinality. If $G-E_{0}$ be $k$-regular or $(k, k+1)$-biregular then from Proposition 1.13, $\gamma\left(G-E_{0}\right)=\gamma^{e}(G-$ $\left.E_{0}\right)$. Hence, $b(G)=b^{e}(G)$.

The exact values of $b_{t}^{e}$ for $L_{n}$ and $b_{t}$ for $D S\left(P_{n}\right)$ are obtained in next two results.

Theorem 2.3: $b_{t}^{e}\left(L_{n}\right)= \begin{cases}1 & ; \text { if } n \equiv 0(\bmod 3) \\ 2 & ; \text { if } n \equiv 2(\bmod 3) \\ 3 & ; \text { otherwise }\end{cases}$

Proof: Let $V\left(L_{n}\right)=\left\{u_{i}, v_{i} / 1 \leq i \leq n\right\}$ be the vertex set with $\left|V\left(L_{n}\right)\right|=2 n$ and $E\left(L_{n}\right)=\left\{e_{i}, e_{i}^{\prime}, f_{i} / 1 \leq i \leq n\right\} \quad$ where $\quad d_{L_{n}}\left(u_{1}\right)=d_{L_{n}}\left(v_{1}\right)=d_{L_{n}}\left(u_{n}\right)=d_{L_{n}}\left(v_{n}\right)=n$ and $d_{L_{n}}\left(u_{i}\right)=d_{L_{n}}\left(v_{i}\right)=3$ for all $i \in\{2,3, \ldots, n-1\}$. Moreover $L_{n}$ is a $(2,3)-$ biregular graph.

To prove the result we consider the following cases:

Case I: For $n \not 1(\bmod 3)$

From Proposition 1.15, there exist $E_{0}=\left\{e_{1}, e_{1}^{\prime}\right\} \subseteq E(G)$. Further $H=L_{n}-E_{0}$ is a graph with two components namely $P_{2}$ and $L_{n-1}$. Then $\gamma_{t}^{e}(H)=\gamma_{t}^{e}\left(P_{2}\right)+\gamma_{t}^{e}\left(L_{n-1}\right)=$ $\gamma_{t}^{e}\left(L_{n}\right)+2$. Hence, $b_{t}\left(L_{n}\right)=b_{t}^{e}\left(L_{n}\right)$.

Case II: For $n \equiv 1(\bmod 3)$

The graph $H$ obtained by removal of three edge namely $e_{n-1}, e_{n}$ and $e_{n}{ }^{\prime}$ for all $i \in\{2,3, \ldots, n-1\}$ from $L_{n}$ is a graph with two components namely $L_{n-1}-e_{n-1}$ and $P_{2}$.

Then $\gamma_{t}^{e}(H)=\gamma_{t}^{e}\left(L_{n-1}-e_{n-1}\right)+\gamma_{t}^{e}\left(P_{2}\right)=\gamma_{t}^{e}\left(L_{n-1}\right)+1+2=\gamma_{t}^{e}\left(L_{n}\right)+1$. Hence, $b_{t}\left(L_{n}\right)=b_{t}^{e}\left(L_{n}\right)$.

Theorem 2.4: $b_{t}\left(D S\left(P_{n}\right)\right)=\left\{\begin{array}{l}1 ; \text { if } n=4 \\ 4 ; \text { if } n>4\end{array}\right.$

Proof: Let $V\left(D S\left(P_{n}\right)\right)=\left\{v_{i} / 1 \leq i \leq n\right\} \cup S_{1} \cup S_{2}$, where $S_{1}=\left\{v_{1}, v_{n}\right\}$ and $S_{2}=\left\{v_{i} / 1 \leq i \leq n-1\right\}$. To obtained $D S\left(P_{n}\right)$ from $P_{n}$, add two new vertices $x$ and $y$ corresponding to $S_{1}$ and $S_{2}$ respectively. Thus $V\left(D S\left(P_{n}\right)\right)=V\left(P_{n}\right) \cup\{x, y\}$ and $E\left(D S\left(P_{n}\right)\right)=E\left(P_{n}\right) \cup\left\{x v_{i} / v_{i} \in S_{1}\right\} \cup\left\{y v_{j} / v_{j} \in S_{2}\right\}$.

To prove the result we consider following cases:

\section{Case I: For $n=4$}

If the graph $H$ is obtained by removal of an edge $v_{2} v_{3}$ from $D S\left(P_{n}\right)$ then $H$ is isomorphic to $C_{6}$. Therefore, $\gamma_{t}(H)=\gamma_{t}\left(C_{6}\right)=4=\gamma_{t}\left(D S\left(P_{4}\right)\right)+1$. Hence $b_{t}\left(D S\left(P_{4}\right)\right)=1$.

\section{Case II: For $n>4$}

The graph $H$ is obtained by removal of four edges namely $e_{1}=v_{2} v_{3}, e_{2}=v_{4} v_{5}$, $e_{3}=y v_{3}$ and $e_{4}=y v_{4}$ from $D S\left(P_{n}\right)$ then $H$ is a graph with two components namely $P_{2}$ and $H_{1}$. Further the minimal total dominating set of $H_{1}$ is $\left\{v_{1}, v_{2}, v_{n-1}, y\right\}$. Then $\gamma_{t}(H)=$ $\gamma_{t}\left(P_{2}\right)+\gamma_{t}\left(H_{1}\right)=2+4=\gamma_{t}\left(D S\left(P_{n}\right)\right)+2$. Hence $b_{t}\left(D S\left(P_{n}\right)\right)=2$ for $n>4$. 
We introduce a concept of total equitable edge critical graph.

Definition 2.5: A graph $G$ is called total equitable domination edge critical or $\gamma_{t}^{e}$-critical graph if the removal of any edge in the graph changes the total equitable domination number. i.e. $\gamma_{t}^{e}(G-e) \neq \gamma_{t}^{e}(G)$ for every edge $e \in E(G)$.

We note that removing an edge from a graph $G$ cannot decrease the total equitable domination number. Hence if $G$ is $\gamma_{t}^{e}$-critical, then $\gamma_{t}^{e}(G-e)>\gamma_{t}^{e}(G)$ for every edge $e \in E(G)$. An edge $e \in E(G)$ is a critical edge of $G$ if $\gamma_{t}^{e}(G-e)>\gamma_{t}^{e}(G)$.

Theorem 2.6: Let $G$ be a $\gamma_{t}^{e}$ - critical graph and $D$ be total equitable dominating set, then the induce subgraph $\langle D\rangle$ is a family of stars.

Proof: Let $D$ be a total equitable dominating set in the $\gamma_{t}^{e}-$ critical graph $G$. Let $e$ be any edge from $\langle D\rangle$. We assume that $\langle D\rangle$ is not a family of stars. Then there exist an edge $e \in D$ such that both terminals of an edge $e$ have degree at least two in $\langle D\rangle$ then $D$ is a total dominating set in $G-e$. Thus $\gamma_{t}^{e}(G-e) \leq|D|=\gamma_{t}^{e}(G)$. This contradicts the graph $G$ is $\gamma_{t}^{e}-$ critical graph (i.e. $\gamma_{t}^{e}(G-e)>\gamma_{t}^{e}(G)$ ). Therefore at least one terminal of an edge $e$ is pendant vertex in $\langle D\rangle$. Hence $\langle D\rangle$ is a family of stars.

We define a family of some trees $\tau$ as: If $T$ is a star $K_{1, n}$ or $T$ is a subdivided star $K_{1, n}^{*}$ then a tree $T$ is member of $\tau(T \in \tau)$.

Theorem 2.7: A tree $T \in \tau$ if and only if $T$ is a $\gamma_{t}^{e}-$ critical.

Proof: First we assume that a tree $T \in \tau$. Let $e$ be any edge of $T$. If one end vertex of $e$ is pendant vertex in $T$ then $\gamma_{t}^{e}(T-e)=\infty$, thus an edge $e$ of $T$ is a critical edge. Therefore we may suppose that both end vertices of an edge $e$ are not pendant in $T$. Then $T$ is a subdivided star by adding pendant edge to each pendent vertex of $T$. If $e$ is an edge from $T$ then $\gamma_{t}^{e}(T-e)=\gamma_{t}^{e}(T)+2$. Therefore $\gamma_{t}^{e}(T-e)=\gamma_{t}^{e}(T)$. Hence $T$ is $\gamma_{t}^{e}-$ critical.

Conversely, suppose that $T=(V(T), E(T))$ is a $\gamma_{t}^{e}-$ critical. Let $D$ be a total equitable dominating set in $T$. By Theorem 2.6, $\langle D>$ is a family of stars and for any edge $e$ of $T$, the set $D$ is not a total equitable dominating set in $T-e$. Then exactly one vertex of $D$ is adjacent to $V(T)-D$ (i.e. $V(T)-D$ is independent set). Thus $T$ is a connected and also $\langle D\rangle$ is a connected graph. Therefore $\langle D\rangle$ is a star. We note that the pendant vertex of $\langle D>$ is adjacent to at least one vertex in $V(T)-D$. Therefore $T$ is a star $K_{1, n}$ or $T$ is a subdivided star $K_{1, n}^{*}$ as $V(T)-D$ is an independent set. Hence $T \in \tau$.

\section{Conclusion}

We have introduced the concept of total equitable bondage number in reference [8]. Here we have explored the same concept. Also proved some characterizations and investigate total equitable bondage number for Ladder $L_{n}$ and degree splitting graph of path $P_{n}$.

\section{Acknowledgment}

The authors are highly thankful to the anonymous referee for kind comments and constructive suggestions on the first draft of this paper. 


\section{References}

1. J. F. Fink, M. S. Jacobson, L. F. Kinch, and J. Roberts, Discrete Math. 86, 47 (1990). https://doi.org/10.1016/0012-365X(90)90348-L

2. V. Swaminathan and K. M. Dharmalingam, J. Math. 35, 191 (2011).

3. G. Deepak, N. D. Soner, and A. Alwaedi, Res. J. Pure Algebra 1, 201 (2011).

4. E. J. Cockayne, R. M. Dawes, and S. T. Hedetniemi, Networks 10, 211 (1980). https://doi.org/10.1002/net.3230100304

5. V. R. Kulli and D. K. Patwari, Vishwa Int. Publications 227 (1991).

6. B. Basavanagoud, V. R. Kulli, and V. V. Teli, J. Computer. Math. Sci. 5, 235 (2014).

7. S. K. Vaidya and A. D. Parmar, J. Comput. Mathematica 1, 98 (2017).

8. S. K. Vaidya and A. D. Parmar, Int. J. Math. Soft Computing 7, 103 (2017). https://doi.org/10.26708/IJMSC.2017.2.7.09

9. S. K. Vaidya and A. D. Parmar, Malaya J. Matematik 6, 375 (2018). https://doi.org/10.26637/MJM0602/0012

10. S. K. Vaidya and A. D. Parmar, On Total and Total Equitable Domination Number of Some Graphs, Thai J. Math. Submitted.

11. S. K. Vaidya and A. D. Parmar, J. Sci. Res. 10, 231 (2018). https://doi.org/10.3329/jsr.v10i3.33940

12. F. Harary, Graph Theory (Addison Wesley, Massachusetts, 1969). https://doi.org/10.21236/AD0705364

13. R. Ponraj and S. Somasundaram, Natl. Acad. Sci. Letters, 27, 275 (2004).

14. F. Hu, Y. Lu, and J. Xu, Discrete Appl. Math. 160, 2408 (2012). https://doi.org/10.1016/j.dam.2012.06.012

15. D. B. West, Introduction to Graph Theory (Prentice Hall, New Delhi, India, 2003).

16. T. W. Haynes, S. T. Hedetniemi, and P. J. Slater, Fundamentals of Domination in Graphs (Marcel Dekker, New York, 1998). 